\title{
Effects of butanedione monoxime on neuromuscular transmission
}

\author{
P.W. Gage, ${ }^{1}$ J.J. McArdle \& D.A. Saint \\ John Curtin School of Medical Research, Australian National University, Canberra, A.C.T. 2601, Australia
}

1 The amplitude of endplate potentials was increased by concentrations of butanedione monoxime (BDM, 5-20 mM) that typically caused muscle paralysis.

2 Although BDM slowed the decay of spontaneous miniature endplate currents, the effect was insuffient to explain most of the large increase in amplitude of endplate potentials.

3 The quantal content of endplate potentials was increased by BDM in a reversible, concentrationdependent manner.

4 The frequency of miniature endplate potentials was not changed by $10 \mathrm{mM}$ BDM in the presence of normal or raised potassium concentrations, indicating that BDM does not change quantal content by a direct effect on calcium channels or on steady-state intracellular calcium concentration.

5 A change in the time course of the extracellularly recorded nerve terminal action potential caused by BDM was similar to the change produced by 4-aminopyridine (4-AP).

6 The increase in quantal content produced by BDM was only slightly reduced in the presence of $1 \mathrm{mM}$ tetraethylammonium (TEA) but was significantly reduced in the presence of 0.5 to $1 \mathrm{mM}$ 4-AP.

7 It was concluded that BDM blocks a 4-AP-sensitive potassium conductance in motor nerve terminals and, by increasing the duration of the action potential in this way, increases evoked transmitter release.

\section{Introduction}

The chemical phosphatase, butanedione monoxime (BDM) has been shown to depress contraction in both skeletal (Mulieri \& Alpert, 1984) and cardiac (Li et al., 1985) muscle. It has been suggested that this effect is related to an action on calcium channels, e.g. block of calcium fluxes across the muscle membrane in response to depolarization (Wiggens et al., 1980) or inhibition of calcium release from the sarcoplasmic reticulum (Fryer et al., 1988). The calcium current in the surface membrane of mammalian skeletal muscle is indeed blocked by BDM (Fryer et al., 1988). Because of the possibility that BDM might also block calcium channels in nerve terminals responsible for transmitter secretion, we have explored its effect on neuromuscular transmission. Far from depressing neuromuscular transmission, BDM caused a large increase in transmitter secreted in response to an action potential; this effect is probably related to an action on potassium channels.

\section{Methods}

The effects of BDM on transmitter secretion were examined in several different nerve-muscle preparations from mice; extensor digitorum longus (EDL), triangularis sterni and phrenic nerve-diaphram. The latter two preparations were used because they are relatively thin and it was hoped that a more homogeneous distribution of drugs amongst the muscle fibres would result. Nerve-muscle preparations were pinned to a block of Sylgard (Dow Corning) in a tissue bath and superfused for $30 \mathrm{~min}$ with a control solution containing either (mM): $\mathrm{NaCl} 150, \mathrm{KCl} 5, \mathrm{CaCl}_{2} 2, \mathrm{MgCl}_{2} 1$, TES buffer 2 (pH 7.4), or $\mathrm{NaCl} 125, \mathrm{NaHCO}_{3} 25, \mathrm{KCl} 5, \mathrm{CaCl}_{2} 2, \mathrm{MgCl}_{2} 1 ; \mathrm{pH}$ 7.4 when bubbled with a gas mixture containing $5 \% \mathrm{CO}_{2}$ and $95 \% \mathrm{O}_{2}$. No obvious differences were observed between results obtained with either of these two control solutions.

\footnotetext{
${ }^{1}$ Present address: Department of Pharmacology, University of Medicine and Dentistry of NJ, 185 South Orange Avenue, Newark, NJ, 07103-2757, U.S.A.
}

Endplate potentials (e.p.ps) were evoked by stimulating the nerve through a suction electrode with pulses of 0.2 to $1 \mathrm{~ms}$ duration. Contraction was suppressed when necessary either by raising the magnesium concentration in the bath solution or by crushing the muscle fibres on either side of the endplate region. Spontaneous miniature endplate potentials (m.e.p.ps) and e.p.ps were recorded with conventional intracellular microelectrodes filled with $3 \mathrm{M} \mathrm{KCl}$. Miniature endplate currents were recorded either by a two electrode point voltage clamp or extracellularly by blunt microelectrodes filled with control solution and pressed against the muscle in the region of a nerve terminal. Endplate currents and nerve terminal action potentials were recorded extracellularly. Experiments were done at room temperature $\left(20-25^{\circ} \mathrm{C}\right)$.

\section{Results}

Addition of BDM to the bath solution at concentrations from 5 to $20 \mathrm{~mm}$ produced a rapid decrease in the twitch tension produced in response to nerve stimulation. However, the drug rarely caused complete depression of twitches within $30 \mathrm{~min}$ in whole muscles, presumably because the concentration attainable in deeper fibres was limited by slow diffusion through the tissue. It was therefore usually necessary to use solutions containing a raised magnesium concentration or to crush muscles on either side of the endplate region in order to obtain completely paralysed preparations. In the presence of BDM, it was possible to record action potentials in muscle fibres in response to nerve stimulation (Figure 1). Such observations indicated that excitation-secretion coupling in the nerve terminal, as well as muscle action potentials, were preserved in preparations paralysed with BDM.

In the presence of BDM, e.p.ps were not only still present but their amplitude was considerably increased, as illustrated in Figure 2. In this preparation, muscle fibres were crushed to prevent contraction before exposure to BDM. E.p.ps recorded in the presence of $10 \mathrm{mM}$ BDM were approximately 5 times larger than control e.p.ps. This effect was readily reversible when the solution containing BDM was washed out of the bath. 


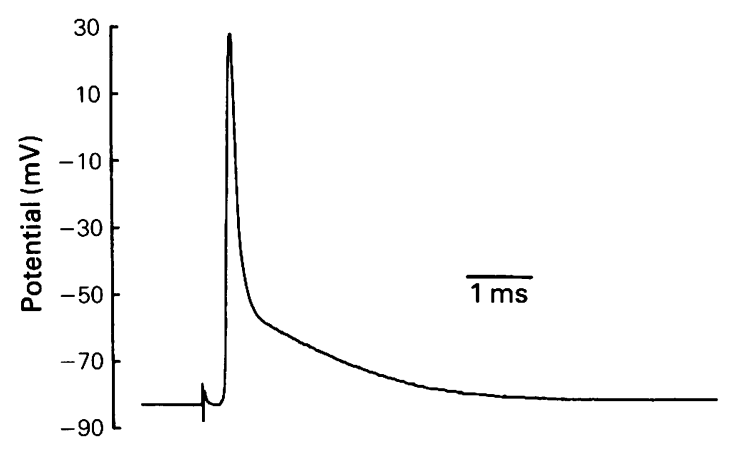

Figure 1 An action potential, evoked by motor nerve stimulation, recorded intracellularly from a surface muscle fibre in a rat extensor digitorum longus (EDL) muscle immobilised with $20 \mathrm{~mm}$ butanedione monoxime (BDM). There was a small amount of movement from deeper fibres but this was insufficient to dislodge the electrode during repeated stimuli.

This increase in e.p.p. amplitude could be due to presynaptic or postsynaptic effects of the drug, e.g. an increase in transmitter release or an increase in the postsynaptic response to acetylcholine. The latter possibility was tested by recording currents generated by single quanta of acetylcholine.

\section{Postsynaptic effect of BDM}

The effect of BDM on miniature endplate currents (m.e.p.cs) is illustrated in Figure 3.

In the presence of BDM $(10 \mathrm{~mm})$, the decay of m.e.p.cs remained exponential but the decay time constant increased on average from $1.2 \mathrm{~ms}$ to $2.2 \mathrm{~ms}$. This effect was readily reversible and could be reproduced several times at the same endplate during successive exposures to the drug (see, for example, Figure 5). This increase in the decay time constant could be responsible for a small part of the increase in e.p.p. amplitude (Gage \& McBurney, 1973), but was not sufficient to account for the 4 to 5 fold increase in e.p.p. amplitude illustrated in Figure 2. The postsynaptic effect of BDM, which may have been due to an interaction with acetylcholinesterase (Wilson \& Ginsberg, 1955), was not investigated further, but was used as a convenient indicator of the presence of BDM at a synapse being investigated.

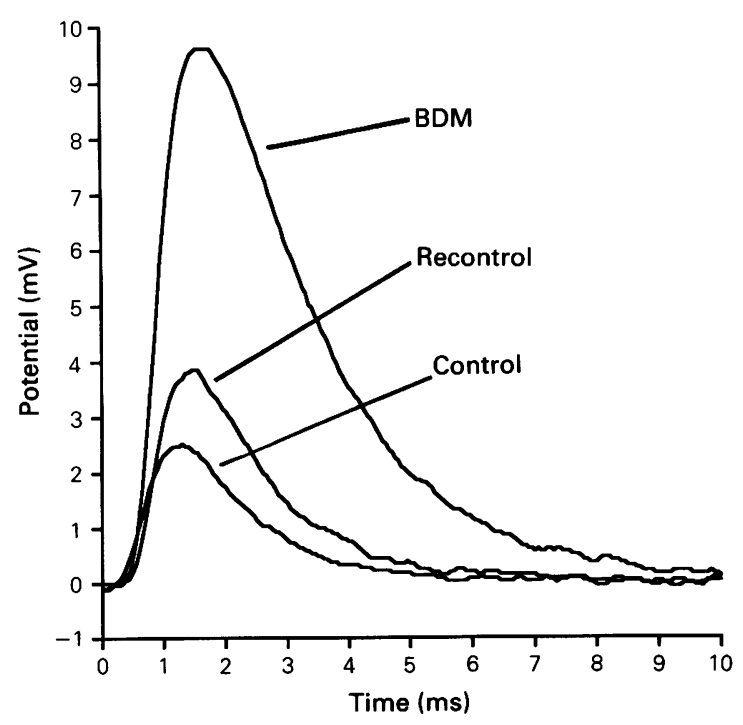

Figure 2 Butanedione monoxime (BDM) increases endplate potential (e.p.p.) amplitude. E.p.ps were recorded in an extensor digitorum longus (EDL) preparation in which the fibres had been crushed to prevent twitching. The bathing solution contained $14 \mathrm{mM}$ magnesium and $2 \mathrm{mM}$ calcium. Traces show a representative e.p.p. before (control), during (BDM) and after (recontrol) exposure to $10 \mathrm{mM}$ BDM.

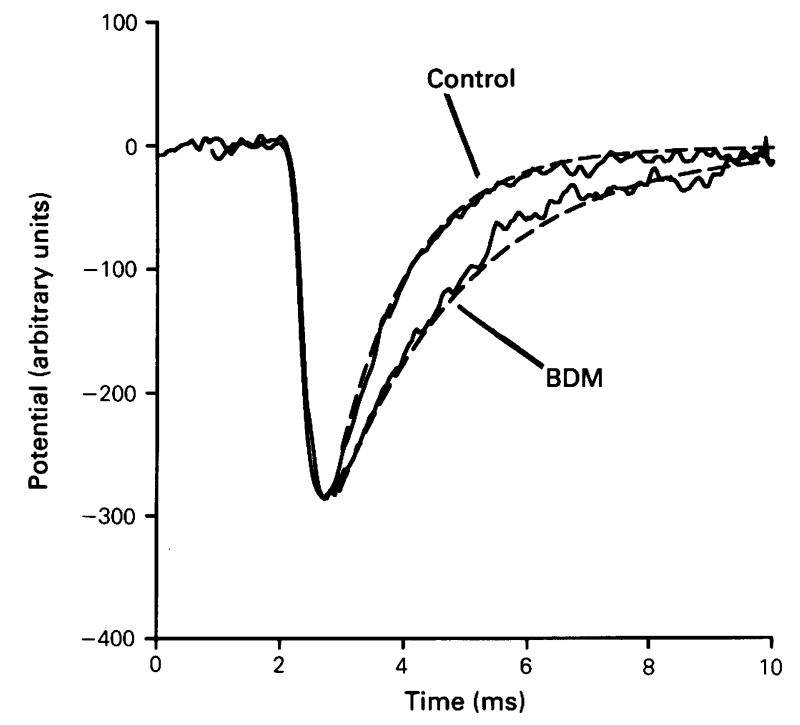

Figure 3 Postsynaptic effect of butanedione monoxime (BDM). Spontaneous miniature endplate currents (m.e.p.cs) were recorded extracellularly at an endplate in a mouse triangularis sterni preparation. The currents shown are averaged m.e.p.cs recorded in control solution $(n=14)$, and in the presence of $10 \mathrm{mM} \mathrm{BDM}(n=14)$. The decays were fitted with single exponentials with time constants of $1.2 \mathrm{~ms}$ and $2.2 \mathrm{~ms}$ in the control and BDM solutions, respectively.

\section{Presynaptic effects of $B D M$}

Quantal content of e.p.ps The increase in the amplitude of e.p.ps (Figure 2) is likely to be due largely to an increase in the quantal content. This is difficult to measure accurately when the quantal content is high because of non-linear summation, which is particularly troublesome in crushed fibre preparations in which the resting membrane potential is usually greatly reduced. The effect of BDM on the quantal content of e.p.ps was measured, therefore, in solutions containing raised magnesium and reduced calcium concentrations, so that quantal content could be estimated from the proportion of 'failures' of e.p.ps in response to presynaptic action potentials (Del Castillo \& Katz, 1954). This method provides an accurate measure of presynaptic changes, uncomplicated by postsynaptic effects, provided that quantal responses are distinguishable from noise.

Concentrations of BDM from 5 to $20 \mathrm{~mm}$ produced increases in the quantal content of e.p.ps. The results shown in Figure 4 were obtained at one junction in a diaphragm preparation, in a solution containing $10 \mathrm{~mm}$ magnesium and $0.5 \mathrm{~mm}$ calcium. The increase in quantal content was proportional to drug concentration over the range examined.

Frequency of m.e.p.ps An increase in resting intracellular calcium concentration could be responsible for this increase in quantal content. If so, BDM would be expected to cause an increase in the frequency of m.e.p.ps. However, when BDM was added at concentrations up to $20 \mathrm{~mm}$ to the normal extracellular solution there was no appreciable effect on the frequency of m.e.p.ps. Even when m.e.p.p. frequency was elevated in a solution containing $15 \mathrm{~mm}$ potassium and $8 \mathrm{~mm}$ calcium, when modulation of calcium channels would be expected to have a large effect on m.e.p.p. frequency (Cooke \& Quastel, 1973), addition of BDM to the extracellular solutions had little effect, as illustrated in Figure 5.

It can be seen that the frequency of m.e.p.ps, measured in $20 \mathrm{~s}$ time bins during addition and removal of $10 \mathrm{mM}$ BDM, showed no abrupt change in the presence of BDM, although the postsynaptic effect, monitored by simultaneous recording of m.e.p.ps, confirmed the presence of BDM at the junction very soon after addition of the drug.

Another possible explanation for the effect of BDM on the quantal content of e.p.ps might be a change in the action 


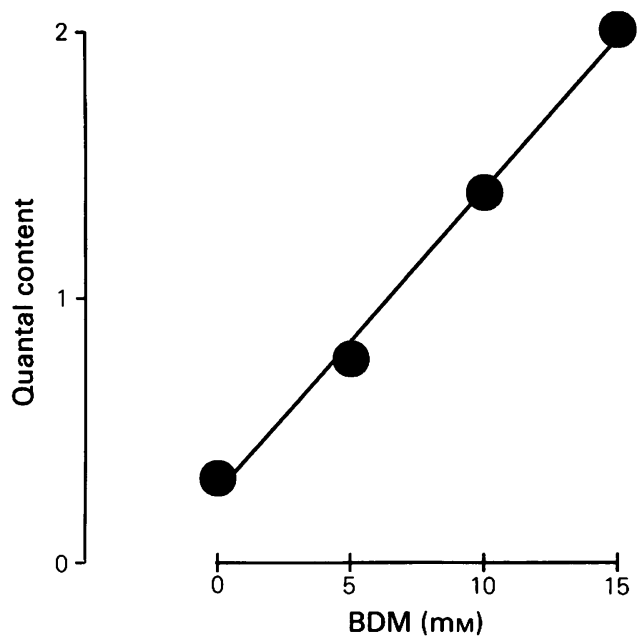

Figure 4 Butanedione monoxime (BDM) increases the quantal content of endplate potentials (e.p.ps) and the effect is concentrationdependent. Quantal content of e.p.ps was measured by the method of failures in a phrenic nerve-diaphragm preparation bathed in a solution containing $10 \mathrm{mM}$ magnesium and $0.3 \mathrm{mM}$ calcium (bicarbonate buffer). The standard errors of the means were smaller than the size of the symbols used.

potential in the presynaptic nerve terminals. Indeed, BDM did produce some change in the time course of the nerve terminal action potential, as can be seen in Figure 6.

The increase in the size of the e.p.c. in $10 \mathrm{mM}$ BDM was accompanied by a delay and broadening of the second part of the presynaptic current. This prolongation is very similar to that observed with drugs that block potassium channels (Burley \& Jacobs, 1981; Brigant \& Mallart, 1982).

In order to explore the possibility that BDM increases quantal content by blocking potassium channels, its effect following exposure to two well characterised potassium channel blockers was examined. In the presence of $1 \mathrm{~mm}$ tetraethylammonium (TEA), a concentration that has been shown to be near maximal in increasing quantal content (Saint $e t$ al., 1987), BDM still caused an increase in quantal content similar to that seen in the absence of TEA. At 7 endplates in which quantal content was depressed in a solution containing $10 \mathrm{~mm}$ magnesium and $0.5 \mathrm{mM}$ calcium, exposure to $10 \mathrm{mM}$ BDM increased quantal content on average by a factor of $6.7 \pm 1.0$ (mean \pm 1 s.e.mean). In the presence of $1 \mathrm{mM}$ TEA, $10 \mathrm{~mm}$ BDM increased quantal content on average by $5.8 \pm 2.0$.

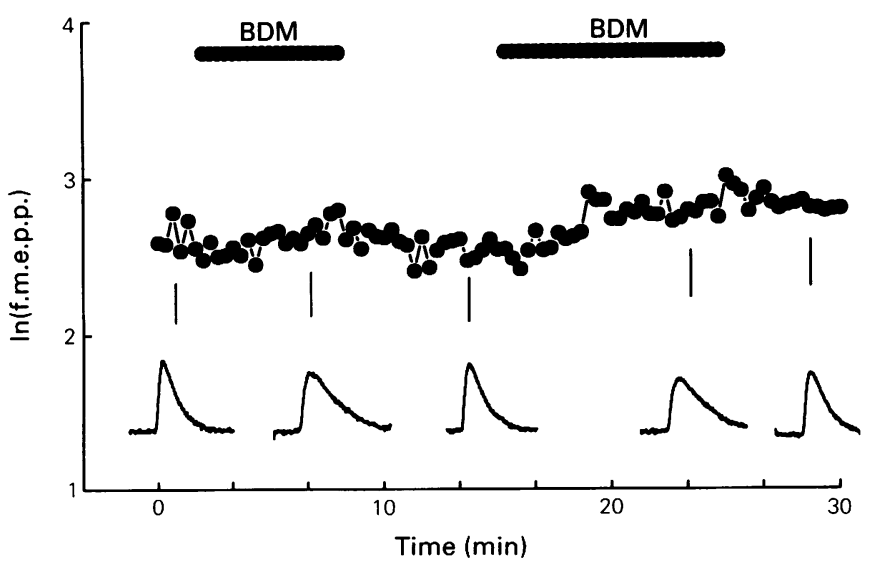

Figure 5 The lack of effect of butanedione monoxime (BDM) on miniature endplate potential (m.e.p.p.) frequency. The frequencies, measured in $20 \mathrm{~s}$ time bins at a single junction in a phrenic nervediaphragm preparation, are plotted semilogarithmically. The bathing solution contained $15 \mathrm{~mm}$ potassium and $8 \mathrm{~mm}$ calcium and the preparation was exposed to a solution containing $10 \mathrm{mM}$ BDM during the times indicated by the horizontal bars. Averaged m.e.p.ps recorded at the times indicated are shown below.

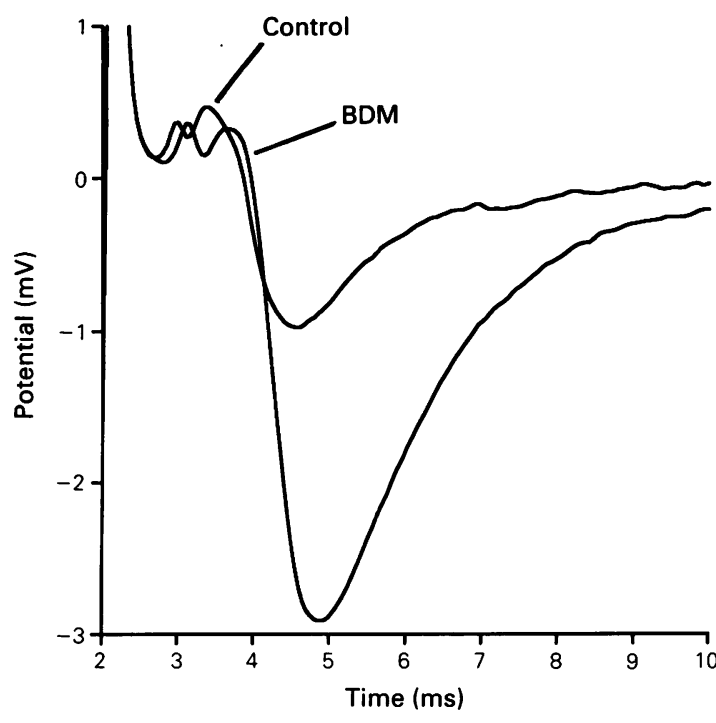

Figure 6 Effect of butanedione monoxime (BDM) on extracellularly recorded nerve terminal action potentials. Endplate currents (e.p.cs) were recorded with an extracellular electrode placed over an endplate in a mouse triangularis sterni preparation. The bathing solution contained $15 \mathrm{~mm}$ magnesium and $2 \mathrm{mM}$ calcium (TES buffer). The addition of BDM produced a change in the time course of the nerve terminal action potential accompanied by a large increase in the amplitude of the e.p.c.

These increases in quantal content in the presence and absence of TEA were not statistically significant at the 5\% level (Student's $t$ test).

In contrast, the effect of BDM on quantal content was clearly depressed in the presence of 4-aminopyridine (4-AP). At 7 junctions in another 3 diaphragm preparations, $10 \mathrm{~mm}$ BDM increased quantal content by a factor of $5.6 \pm 1.5$. At another 7 junctions in the same 3 preparations, $10 \mathrm{~mm}$ BDM increased quantal content by a factor of only $1.5 \pm 0.2$ in the presence of $0.5 \mathrm{mM}$ 4-AP. This depression of the effect of BDM by 4-AP was significant at the $5 \%$ level (Student's $t$ test). The occlusion of the effects of 4-AP and BDM on quantal content was demonstrated clearly at 2 other junctions which survived multiple solution changes (Table 1). Before exposure to 4-AP, $10 \mathrm{mM}$ BDM increased quantal content by factors of 4.9 and 4

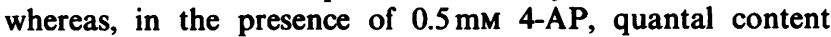
increased by factors of only 1.6 and 1.3 .

\section{Discussion}

The results indicate that BDM, at concentrations that effectively depress muscle contraction, does not depress transmitter secretion from motor nerve terminals. It can be concluded therefore that BDM does not depress the calcium current

Table 1 Reduction in the effect of butanedione monoxime (BDM) on quantal content in the presence of $0.5 \mathrm{mM} 4$ aminopyridine (4-AP) in two phrenic nerve-diaphragm preparations

$\begin{array}{lllll} & \text { Control } & \text { BDM } & \begin{array}{l}\text { Control } \\ +4-A P\end{array} & B D M+4-A P \\ \text { Prep 1 } & 0.052 & 0.257 & 2.72 & 4.48 \\ \text { Ratio } & & 4.9 & & 1.6 \\ \text { Prep 2 } & 0.098 & 0.391 & 3.97 & 5.31 \\ \text { Ratio } & & 4.0 & & 1.3\end{array}$

Solutions contained $10 \mathrm{~mm}$ magnesium and $0.3 \mathrm{~mm}$ calcium. In control and the BDM $(10 \mathrm{mM})$ solution, quantal content was calculated from failures whereas, in solutions containing 4-AP, quantal content was calculated from the ratio of average e.p.p. and m.e.p.p. amplitudes. 
responsible for transmitter secretion. In fact, BDM produces a large increase in quantal content of e.p.ps without affecting the frequency of m.e.p.ps. The latter observation makes it unlikely that BDM alters intracellular calcium concentration by changing the characteristics of calcium channels or the storage of intracellular calcium. On the other hand, prolongation of the presynaptic action potential would be expected to increase evoked but not spontaneous transmitter release. Indirect evidence that BDM produces the increase in quantal content in this way is presented: BDM alters the time course of the nerve terminal action potential and is less effective in increasing quantal content in the presence of 4-AP. In view of

\section{References}

BRIGANT, J.L. \& MALLART, A. (1982). Presynaptic currents in mouse motor endings. J. Physiol., 333, 619-636.

BURLEY, E.S. \& JACOBS, R.S. (1981). Effects of 4-aminopyridine on nerve terminal action potentials. J. Pharmacol. Exp. Ther., 219, 268-273.

COOKE, J.D. \& QUASTEL, D.M.J. (1973). Transmitter release by mammalian motor nerve terminals in response to focal polarisation. $J$. Physiol., 228, 377-405.

DEL CASTILLO, J. \& KATZ, B. (1954). Quantal components of the endplate potential. J. Physiol., 124, 560-573.

FRYER, M. W., GAGE, P. W., NEERING, I.R., DULHUNTY, A.F. \& LAMB G.D. (1988). Paralysis of skeletal muscle by butanedione monoxime, a chemical phosphatase. Pflügers Arch., 411, 76-79.

GAGE, P.W. \& MCBURNEY, R.N. (1973). An analysis of the relationship between the current and potential generated by a quantum of acetylcholine in muscle fibres without transverse tubules. J. Memb. Biol., 12, 247-272. these observations, it seems very likely that most of the increase in quantal content caused by BDM is related to a depression of a potassium conductance in the motor nerve terminal that is sensitive to 4-AP. It may be that this potassium channel is functionally depressed when dephosphorylated.

Whatever the mode of action of BDM on transmitter release, the persistence of quantal secretion at neuromuscular junctions in which muscle fibres are immobilised provides a new preparation for quantitative studies of synaptic transmission and electrical activity, such as asymmetric charge movement in skeletal muscle fibres.

LI, T., SPERELAKIS, N., TENEICK, R.E. \& SOLARO, J. (1985). Effects of diacetyl monoxime on cardiac excitation-contraction coupling. $J$. Pharmacol. Exp. Ther., 232, 688-695.

MULERI, L.A. \& ALPERT, N.R. (1984). Differential effects of 2,3-butanedione monoxime (BDM) on activation and contraction. Biophys. J., 45, 47a.

SAINT, D.A., QUASTEL, D.M.J. \& GUAN, Y-Y. (1987). Multiple potassium conductances at the mammalian motor nerve terminal. Pflïgers Arch., 410, 408-412.

WIGGENS, J.R., REISER, J., FITZPATRICK, D.F. \& BERGEY, J.L. (1980). Inotropic actions of diacetyl monoxime in cat ventricular muscle. J. Pharamcol. Exp. Ther., 212, 217-224.

WILSON, I.B. \& GINSBERG, S. (1955). A powerful reactivator of alkylphosphate-inhibited acetylcholinesterase. Biochim. Biophys. Acta, 18, 168-175. 